PROCEEDINGS OF THE

AMERICAN MATHEMATICAL SOCIETY

Volume 125, Number 5, May 1997, Pages 1419-1424

S 0002-9939(97)03838-0

\title{
A NEW PROOF OF THE TWO WEIGHT NORM INEQUALITY FOR THE ONE-SIDED FRACTIONAL MAXIMAL OPERATOR
}

\author{
DAVID CRUZ-URIBE, SFO
}

(Communicated by J. Marshall Ash)

\begin{abstract}
We give a new proof of the two weight norm inequality for the one-sided, fractional maximal operator, $M_{\alpha}^{+}$, simplifying the original proof of Martín-Reyes and de la Torre.
\end{abstract}

\section{INTRODUCTION}

In [1], Andersen and Sawyer introduced the one-sided fractional maximal operators

$$
M_{\alpha}^{+} f(x)=\sup _{t>0} \frac{1}{t^{1-\alpha}} \int_{x}^{x+t}|f| d y \quad \text { and } \quad M_{\alpha}^{-} f(x)=\sup _{t>0} \frac{1}{t^{1-\alpha}} \int_{x-t}^{x}|f| d y,
$$

$0<\alpha<1$, in order to study the weighted norm inequalities for the RiemannLiouville and Weyl fractional integral operators. Using complex interpolation, they proved one-weight norm inequalities for $M_{\alpha}^{+}$and $M_{\alpha}^{-}$. In [3], Martín-Reyes and de la Torre answered a question of Andersen and Sawyer by giving a geometric proof of a two-weight norm inequality for $M_{\alpha}^{+}$. (The same result holds, mutatis mutandis, for $M_{\alpha}^{-}$.)

Theorem 1.1. For non-negative weights $u$ and $v$, and for $1<p \leq q$, the following are equivalent:

1. There exists a constant $C$ such that, for every function $f$ in $L^{p}(v)$,

$$
\left(\int_{\mathbb{R}}\left(M_{\alpha}^{+} f\right)^{q} u d x\right)^{1 / q} \leq C\left(\int_{\mathbb{R}}|f|^{p} v d x\right)^{1 / p}
$$

2. The pair $(u, v)$ satisfy the $\left(S_{p q \alpha}^{+}\right)$condition: there exists a constant $C$ such that for every interval $I=[a, b]$ for which $u((-\infty, a))>0$,

$$
\left(\int_{I} M_{\alpha}^{+}\left(\sigma \chi_{I}\right)^{q} u d x\right)^{1 / q} \leq C\left(\int_{I} \sigma d x\right)^{1 / p}<\infty
$$

where $\sigma=v^{1-p^{\prime}}$.

Received by the editors August 29, 1995 and, in revised form, October 25, 1995 and November 16, 1995.

1991 Mathematics Subject Classification. Primary 42B25.

Key words and phrases. One-sided fractional maximal operator, weighted norm inequalities.

(C) 1997 American Mathematical Society 
Their proof involved proving a weighted norm inequality for a dyadic variant of the fractional maximal operator, $M_{\alpha D}^{+}$, using a dyadic version of the Sawyer condition, $\left(S_{p q \alpha D}^{+}\right)$, and then showing that these were equivalent to $M_{\alpha}^{+}$and $\left(S_{p q \alpha}^{+}\right)$.

The purpose of this paper is to give a new proof of Theorem 1.1, one which eliminates the dyadic maximal operator. We do this by adapting the proof in the dyadic case using special covering properties of $\mathbb{R}$ and the continuity properties of the maximal operator. We believe that these techniques will be useful in proving other norm inequalities for maximal operators on $\mathbb{R}$.

The paper is organized as follows: Section 2 contains three lemmas and some remarks on their applicability, and Section 3 contains the actual proof. Throughout, all functions are assumed to be measurable, $C$ denotes a positive constant whose value may be different at each appearance, $p^{\prime}=p /(p-1)$ is the conjugate exponent of $p$, and $0<\alpha<1$. Given a Borel set $E$ and a function $w$, let $|E|$ denote the Lebesgue measure of $E$ and $w(E)=\int_{E} w d x$.

\section{Preliminary Results}

Lemma 2.1. Let $f$ be a non-negative, bounded, upper semicontinuous function of compact support. Then $M_{\alpha}^{+} f$ is continuous.

Proof. Since $M_{\alpha}^{+} f$ is always lower semicontinuous, it will suffice to show that it is upper semicontinuous at each point $x \in \mathbb{R}$. We will show this by contradiction: suppose that there exist an $\epsilon>0$, a point $x_{0}$ and a sequence of points $\left\{x_{n}\right\}$ converging to $x_{0}$ such that $M_{\alpha}^{+} f\left(x_{n}\right)>M_{\alpha}^{+} f\left(x_{0}\right)+\epsilon$. For each $n$ there exists an interval $J_{n}$ whose left endpoint is $x_{n}$ such that

$$
\frac{1}{\left|J_{n}\right|^{-\alpha}} \int_{J_{n}}|f| d x>M_{\alpha}^{+} f\left(x_{0}\right)+\epsilon .
$$

Let the support of $f$ be contained in the (finite) interval $I$, and let $K$ be a finite, open interval containing $I$ and the $x_{n}$ 's, $n \geq 0$. Then we may assume that each $J_{n}$ is contained in $K$. Therefore, after passing to a subsequence we may assume that the $J_{n}$ 's converge to a possibly degenerate interval $J$ whose left endpoint is $x_{0}$. If $|J|>0$ then inequality (1) implies that

$$
M_{\alpha}^{+} f\left(x_{0}\right) \geq \frac{1}{|J|^{1-\alpha}} \int_{J}|f| d x \geq M_{\alpha}^{+} f\left(x_{0}\right)+\epsilon,
$$

a contradiction. If $J=\left\{x_{0}\right\}$ then for $n$ sufficiently large, by the upper semicontinuity of $f, f(x)-f\left(x_{0}\right)<\epsilon$ for all $x \in J_{n}$. Therefore,

$$
\frac{1}{\left|J_{n}\right|^{1-\alpha}} \int_{J_{n}} f(x) d x<\left|J_{n}\right|^{\alpha}\left(f\left(x_{0}\right)+\epsilon\right) .
$$

If we combine this with inequality (1) and take the limit as $n$ tends to infinity, we get (since $f$ is bounded) that $0 \geq M_{\alpha}^{+} f\left(x_{0}\right)+\epsilon$, which is again a contradiction. Hence $M_{\alpha}^{+} f$ is upper semicontinuous at each point, and we are done.

It is worth noting that this lemma is not true if $\alpha=0$. A simple counter-example is given by the characteristic function of $[0,1]$. (I am grateful to Juha Kinnunen for pointing this out to me.)

The next lemma is due to Jesus Aldaz; the proof is in Bliedtner and Loeb [2]. 
Lemma 2.2. If $\mu$ is a finite Borel measure on $\mathbb{R}$, and if $\mathcal{I}$ is an arbitrary collection of non-degenerate intervals, then for each $\delta>0$ there exists a finite subcollection, $\mathcal{I}_{\delta}$, of disjoint intervals in $\mathcal{I}$ such that

$$
\mu\left(\bigcup_{I \in \mathcal{I}} I\right) \leq(2+\delta) \sum_{I_{k} \in \mathcal{I}_{\delta}} \mu\left(I_{k}\right)
$$

Below we will want to apply Lemma 2.2 with the measure $u d x$, where $(u, v)$ satisfies the $\left(S_{p q \alpha}^{+}\right)$condition. To do this, we need $u$ to be locally integrable and the intervals to be contained in some compact set. However, if $I=[a, b]$ is an interval such that $u((-\infty, a))>0$, and if there exists an interval $J=[b, c]$ such that $\sigma(J)>0$, then for all $x \in I$,

$$
M_{\alpha}^{+}\left(\sigma \chi_{I \cup J}\right)(x) \geq \sigma(J) /(c-a)^{1-\alpha} .
$$

Hence, by the $\left(S_{p q \alpha}^{+}\right)$condition applied to $I \cup J$,

$$
u(I) \leq C \sigma(I)^{q / p}(c-a)^{1-\alpha} / \sigma(J)<\infty .
$$

If no such $J$ exists, then $\sigma \equiv 0$ on $[b, \infty)$, so $v \equiv \infty$ on the same set. But if $f \in L^{p}(v)$, then $f \equiv 0$ on $[b, \infty)$.

Below, we will apply Lemma 2.2 to closed intervals contained in an open set $O_{k}$. In $O_{k}, M_{\alpha}^{+} f>0$, so $f$ cannot be identically zero to the right of these intervals. Further, by the definition of $O_{k}, u$ is not identically zero to the left of these intervals. Finally, since we will also be assuming that $f$ has compact support, the intervals will be contained in some compact set. Hence Lemma 2.2 is applicable.

The last lemma is an extension of a result of Muckenhoupt [4] for Lebesgue measure. The proof of the extension is identical to his proof and so is omitted.

Lemma 2.3. Let $\mu$ be a Borel measure, $f$ a function, and $\left\{I_{\beta}\right\}$ a collection of intervals, all contained in some interval $I$, with the property that

$$
\int_{I_{\beta}} f d \mu \geq N \mu\left(I_{\beta}\right)
$$

If $J=\bigcup_{\beta} I_{\beta}$ then

$$
\int_{J} f d \mu \geq(N / 2) \mu(J)
$$

If $(u, v)$ satisfy the $\left(S_{p q \alpha}^{+}\right)$condition, then $\sigma d x=v^{1-p^{\prime}} d x$ is a Borel measure.

\section{Proof of Theorem 1.1}

To show that the $\left(S_{p q \alpha}^{+}\right)$condition is necessary for inequality (1) of Theorem 1.1 to hold, first suppose that there is some interval $I=[a, b]$ such that $u((-\infty, a))>0$ but $\sigma(I)=\infty$. Equivalently, the function $v^{-1} \chi_{I}$ is not in $L^{p^{\prime}}(v)$, so there exists a function $f$ in $L^{p}(v)$ such that

$$
\infty=\int_{I} f v^{-1} v d x=\int_{I} f d x
$$

Then for all $x \in J, M_{\alpha}^{+} f(x)=\infty$, which contradicts inequality (1). The rest of the $\left(S_{p q \alpha}^{+}\right)$condition follows if we substitute $f=\sigma \chi_{I}$ into the norm inequality.

To prove that the $\left(S_{p q \alpha}^{+}\right)$condition is sufficient, we follow the outline of the proof of Martín-Reyes and de la Torre [3], which in turn is based on a proof by Sawyer [6]. Let $f$ be in $L^{p}(v)$; we will first consider the special case where $f$ is a non-negative, 
bounded, upper semicontinuous function of compact support. By Lemma $2.1, M_{\alpha}^{+} f$ is continuous. Further, since the set $\left\{x: M_{\alpha}^{+} f(x)=\lambda\right\}$ has positive measure for at most a countable number of $\lambda$, by multiplying $f$ by a suitable constant we may assume without loss of generality that the sets $\left\{x: M_{\alpha}^{+} f(x)=2^{k}\right\}$ have measure zero for all integers $k$.

Let $a=\sup \{x: u((-\infty, x))=0\}$. For each integer $k$ define the set $O_{k}=\{x$ : $\left.2^{k}<M_{\alpha}^{+} f(x)<2^{k+1}\right\} \cap(a, \infty)$. Since $M_{\alpha}^{+} f$ is continuous, it follows that each $O_{k}$ is open, and the set $\mathbb{R} \backslash \bigcup_{k} O_{k}$ has measure zero. For each $x \in O_{k}$, there exists an open interval $J_{x k}=\left(x, t_{x}\right)$ such that

$$
2^{k}<\frac{1}{\left|J_{x k}\right|^{1-\alpha}} \int_{J_{x k}} f d y<2^{k+1}
$$

We claim that there exists a point $s_{x} \in J_{x k}$ such that if $y \in I_{x k}=\left[x, s_{x}\right]$ then

$$
\frac{1}{\left|J_{x k}\right|^{1-\alpha}} \int_{J_{x k}} \sigma d y \leq 2 M_{\alpha}^{+}\left(\sigma \chi_{J_{x k}}\right)(y) .
$$

If $M_{\alpha}^{+}\left(\sigma \chi_{J_{x k}}\right)(x)=0$ then this is immediate. If it is positive, then since $M_{\alpha}^{+}\left(\sigma \chi_{J_{x k}}\right)$ is lower semicontinuous, we can find $s_{x}$ such that $M_{\alpha}^{+}\left(\sigma \chi_{J_{x k}}\right)(y)$ is also positive for $y \in I_{x k}$. By the continuity of the integral, the desired inequality holds if we take $s_{x}$ sufficiently close to $x$. Finally, since $O_{k}$ is open we may take $s_{x}$ so that $I_{x k} \subset O_{k}$.

The union of the $I_{x k}$ 's is $O_{k}$. Therefore, by Lemma 2.2 and the remarks following it, there exists a finite, disjoint collection of intervals $\left\{I_{j k}\right\}_{j=1}^{n_{k}}$ such that

$$
u\left(O_{k}\right) \leq 3 \sum_{j=1}^{n_{k}} u\left(I_{j k}\right) .
$$

Since the sets $O_{k}$ are disjoint, the intervals $I_{j k}$ are pairwise disjoint for all $j$ and $k$.

Using inequalities (2) and (4), we can now make the following estimate:

$$
\begin{aligned}
\int_{\mathbb{R}}\left(M_{\alpha}^{+} f\right)^{q} u d x & =\sum_{k} \int_{O_{k}}\left(M_{\alpha}^{+} f\right)^{q} u d x \\
& \leq \sum_{k} u\left(O_{k}\right) 2^{q(k+1)} \\
& \leq C \sum_{j, k} u\left(I_{j k}\right) 2^{q k} \\
& \leq C \sum_{j, k} u\left(I_{j k}\right)\left(\frac{1}{\left|J_{j k}\right|^{1-\alpha}} \int_{J_{j k}} f d x\right)^{q} \\
& =C \sum_{j, k} u\left(I_{j k}\right)\left(\frac{1}{\left|J_{j k}\right|^{1-\alpha}} \int_{J_{j k}} \sigma d x\right)^{q}\left(\frac{\int_{J_{j k}}(f / \sigma) \cdot \sigma d x}{\int_{J_{j k}} \sigma d x}\right)^{q}
\end{aligned}
$$

Define the measure $\omega$ on $X=\mathbb{N} \times \mathbb{Z}$ by

$$
\omega(j, k)=u\left(I_{j k}\right)\left(\frac{1}{\left|J_{j k}\right|^{1-\alpha}} \int_{J_{j k}} \sigma d x\right)^{q}
$$


if $j \leq n_{k}$, and $\omega(j, k)=0$ if $j>n_{k}$. Also define the operator $T$ by

$$
T h(j, k)=\frac{\int_{J_{j k}}|h| \sigma d x}{\int_{J_{j k}} \sigma d x} .
$$

Then, following the argument of Sawyer, to get the desired norm inequality it will suffice to show that $T$ is a bounded operator from $L^{p}(\sigma)$ into $L^{q}(X, \omega)$. Since $T$ is bounded on $L^{\infty}$, by Marcinkiewicz interpolation it will suffice to show that $T$ is weak-type $(1, q / p)$ : that is, for each $\lambda>0$

$$
\sum_{(j, k) \in E_{\lambda}} u\left(I_{j k}\right)\left(\frac{1}{\left|J_{j k}\right|^{1-\alpha}} \int_{J_{j k}} \sigma d x\right)^{q} \leq C\left(\frac{1}{\lambda} \int_{\mathbb{R}}|h| \sigma d x\right)^{q / p},
$$

where $E_{\lambda}=\{(j, k) \in X: T h(j, k)>\lambda\}$. If $(j, k) \in E_{\lambda}$, then

$$
\int_{J_{j k}}|h| \sigma d x>\lambda \int_{J_{j k}} \sigma d x .
$$

Let $G_{\lambda}$ be the union of all such $J_{j k}$ 's. Then, since the $J_{j k}$ 's are open, $G_{\lambda}$ is the union of a countable number of disjoint open intervals $J_{i}$. By Lemma 2.3, for each $i$

$$
\int_{J_{i}}|h| \sigma d x>\frac{\lambda}{2} \int_{J_{i}} \sigma d x .
$$

Since $I_{j k} \subset J_{j k}$, each $I_{j k}$ is contained in exactly one interval $J_{i}$. (Here we ignore the left endpoints of the $I_{j k}$ 's since they form a set of measure zero.) Therefore, by inequalities (3) and (5) and the $\left(S_{p q \alpha}^{+}\right)$condition, since $q / p \geq 1$, and since the $I_{j k}$ 's and the $J_{i}$ 's are disjoint,

$$
\begin{aligned}
\sum_{(j, k) \in E_{\lambda}} u\left(I_{j k}\right)\left(\frac{1}{\left|J_{j k}\right|^{1-\alpha}} \int_{J_{j k}} \sigma d x\right)^{q} & \leq 2 \sum_{(j, k) \in E_{\lambda}} \int_{I_{j k}} M_{\alpha}^{+}\left(\sigma \chi_{J_{j} k}\right)^{q} u d x \\
& \leq 2 \sum_{i} \sum_{I_{j k} \subset J_{i}} \int_{I_{j k}} M_{\alpha}^{+}\left(\sigma \chi_{J_{i}}\right)^{q} u d x \\
& \leq 2 \sum_{i} \int_{J_{i}} M_{\alpha}^{+}\left(\sigma \chi_{J_{i}}\right)^{q} u d x \\
& \leq C \sum_{i}\left(\int_{J_{i}} \sigma d x\right)^{q / p} \\
& \leq C \sum_{i}\left(\frac{1}{\lambda} \int_{J_{i}}|h| \sigma d x\right)^{q / p} \\
& \leq C\left(\frac{1}{\lambda} \int_{\mathbb{R}}|h| \sigma d x\right)^{q / p} .
\end{aligned}
$$

This completes the special case. To complete the proof, take any $f \in L^{p}(v)$; then by the Vitali-Carathéodory theorem, there exists an increasing sequence $\left\{f_{n}\right\}$ of non-negative, bounded, upper semicontinuous functions of compact support which converge to $|f|$. (See, for example, Rudin [5, p. 57].) By the monotone convergence theorem, $M_{\alpha}^{+} f_{n}$ increases pointwise to $M_{\alpha}^{+} f$. Therefore, again by the monotone 
convergence theorem,

$$
\begin{aligned}
\left(\int_{\mathbb{R}}\left(M_{\alpha}^{+} f\right)^{q} u d x\right)^{1 / q} & =\lim _{n \rightarrow \infty}\left(\int_{\mathbb{R}}\left(M_{\alpha}^{+} f_{n}\right)^{q} u d x\right)^{1 / q} \\
& \leq \lim _{n \rightarrow \infty} C\left(\int_{\mathbb{R}} f_{n}^{p} v d x\right)^{1 / p} \\
& =C\left(\int_{\mathbb{R}}|f|^{p} v d x\right)^{1 / p}
\end{aligned}
$$

\section{ACKNOWLEDGMENT}

I want to thank F. Martín-Reyes and A. de la Torre for their hospitality, and my wife Gabrielle for her patient generosity; without either this paper would not have been possible.

\section{REFERENCES}

[1] K. F. Andersen and E. T. Sawyer, Weighted norm inequalities for the Riemann-Liouville and Weyl fractional integral operators, Trans. Amer. Math. Soc. 308 (1988), 547-557. MR 89h:26006

[2] J. Bliedtner and P. Loeb, A reduction technique for limit theorems in analysis and probability theory, Ark. Mat. 30 (1992), 25-43. MR 93k:28005

[3] F. J. Martín-Reyes and A. de la Torre, Two weight norm inequalities for fractional one-sided maximal operators, Proc. Amer. Math. Soc. 117 (1993), 483-489. MR 94b:42010

[4] B. Muckenhoupt, Weighted norm inequalities for the Hardy maximal function, Trans. Amer. Math. Soc. 165 (1972), 207-226. MR 45:4261

[5] W. Rudin, Real and Complex Analysis, McGraw-Hill, New York, 1974. MR 49:8783

[6] E. T. Sawyer, A characterization of a two-weight norm inequality for maximal operators, Studia Math. 75 (1982), 1-11. MR 84i:42032

Department of Mathematics, Trinity College, Hartford, Connecticut 06106-3100

E-mail address: david.cruzuribe@mail.trincoll.edu 